\title{
Risk of Readmission and Mortality Following Hospitalization with Hypercapnic Respiratory Failure
}

\author{
Amber J. Meservey ${ }^{1} \cdot$ Michael C. Burton $^{1} \cdot$ Jeffrey Priest ${ }^{1} \cdot$ Charlotte C. Teneback $^{1} \cdot$ Anne E. Dixon $^{1,2}$ (I)
}

Received: 22 August 2019 / Accepted: 2 December 2019 / Published online: 11 December 2019

(c) Springer Science+Business Media, LLC, part of Springer Nature 2019

\begin{abstract}
Purpose Hypercapnic respiratory failure (HRF) is a frequent cause of hospitalization and a common comorbidity in hospitalized patients. There are few studies addressing what factors might predict poor outcomes in this patient population. The purpose of the current study was to investigate characteristics and outcomes of patients hospitalized with HRF.

Methods A study of patients $\geq 18$ years admitted with HRF in a 1-year period. Patients with limited life expectancy related to other conditions, and those with a non-respiratory cause of HRF, were excluded.

Results 202 subjects met eligibility criteria: $24 \%$ had a diagnosis of obstructive sleep apnea, $6 \%$ obesity hypoventilation, $46 \%$ chronic obstructive pulmonary disease, and $10 \%$ asthma. Fifteen (7\%) died during the index admission. Forty-one patients (23\%) were readmitted within 30 days: peripheral vascular disease [adjusted odds ratio (aOR) 4.78, CI 1.45-15.74] and tachycardia (aOR 2.97, CI 1.22-7.26) were associated with an increased risk of readmission. Sixty-six patients (36\%) died after discharge. Risk of death was increased in older patients (aOR 1.32, CI 1.13-1.54 per 5 years), those with peripheral vascular disease (aOR 12.56, CI 2.35-67.21), higher Charlson co-morbidity index (aOR 1.39, CI 1.09-1.76), use of home oxygen (aOR 4.03, CI 1.89-8.57), and those who had been readmitted (aOR 3.07, CI 1.46-6.43).

Conclusions Hospitalization for HRF is associated with a high morbidity and mortality. Our observation that home oxygen use was associated with increased mortality suggests that oxygen use could be a risk factor for death in patients with HRF.
\end{abstract}

Keywords Hypercapnic respiratory failure $\cdot$ Respiratory failure $\cdot$ Hypercapnia $\cdot$ Non-invasive ventilation $\cdot$ Oxygen therapy

\section{Introduction}

Hypercapnic respiratory failure (HRF), a state of reduced alveolar ventilation with subsequent respiratory acidosis, is often caused by common respiratory diseases such as chronic obstructive pulmonary disease (COPD), obesity hypoventilation syndrome, obstructive sleep apnea (OSA) and the overlap syndrome of COPD with OSA $[1,2]$. The prevalence of these diseases is increasing significantly [3-6]. Because of the increasing prevalence of these underlying conditions, HRF is a common reason for admission and a frequent co-morbidity among hospitalized patients. Despite

Anne E. Dixon

anne.dixon@uvmhealth.org

1 University of Vermont, Burlington, USA

2 University of Vermont, Given D209, 89 Beaumont Avenue, Burlington, VT 05405, USA this, there are few studies addressing outcomes of this patient population.

Patients hospitalized with HRF might represent a patient population with a particularly high risk of mortality and readmission. There have been a number of studies examining outcomes of patients treated with non-invasive ventilation (NIV) for HRF. Ahmed and colleagues reported 53\% of patients treated with NIV for acute HRF died during the index admission [7]. A study of patients discharged from the intensive care unit following an episode of HRF reported that $46 \%$ of patients were readmitted, and $17 \%$ died within 12 months [1]. Morbidity appears particularly high following discharge among COPD patients treated as inpatients with NIV: one study reported $80 \%$ readmitted and $49 \%$ dead within 12 months [8], another found $40 \%$ readmitted and $11 \%$ dead [9]. These studies report high mortality and morbidity, particularly among patients with COPD, but only included patients treated with NIV.

Patients with hypercapnia often have multiple comorbidities related to obesity and tobacco use, and so not all patients 
with HRF will have this as the primary cause of their hospitalization. Many patients with chronic lung disease and/or sleep disordered breathing may be admitted with pneumonia or heart disease, which are also common in this population; hypercapnia in patients hospitalized with pneumonia and heart failure has been associated with adverse outcomes [10-13], and so outcomes in the full spectrum of patients with HRF is of interest.

The purpose of the current study was to investigate outcomes of patients hospitalized with a diagnosis of HRF, rather than just those identified for treatment with NIV. This search strategy was designed to identify all participants hospitalized with HRF, not just those requiring some form of ventilator support. We hypothesized these patients would have a high risk of adverse outcomes, and so identifying such patients might identify a high risk population for future targeted interventions. Of particular interest was investigating how co-morbidities affected patient outcomes. We investigated the mortality rate during the index admission, the 30 day readmission rate, and the late mortality following the index admission.

\section{Methods}

We identified patients $\geq 18$ years admitted with a diagnosis of HRF identified by ICD 10 diagnostic codes [J96.02 (acute HRF), J96.22 (acute and chronic respiratory failure with hypercapnia), J96.92 (respiratory failure unspecified with hypercapnia), J96.12 (chronic respiratory failure with hypercapnia), E66.2 (morbid obesity with hypoventilation)] in the calendar year 2016. This search strategy was designed to identify all participants hospitalized with HRF, not just those requiring some form of ventilator support. Records were reviewed by three investigators (AM, MB and AED), who excluded patients found to have advanced cancer, trauma, acute stroke/seizure, cardiac arrest prior to admission, advanced neurological disorder, or serious nonpulmonary illness (other than HRF) that in the opinion of the investigator would limit life expectancy. We used the Charlson co-morbidity index as a measure of chronic illness and risk of mortality, as updated by Quan et al. [14, 15].

Data abstracted from the electronic medical record included demographics, co-morbidities, hospital course and course after discharge. Data were entered into a RedCap database hosted by the University of Vermont [16].

\section{Statistical Methods}

We calculated frequencies and means of demographic characteristics and other data from participants' electronic medical record, including those who died during the index admission, those readmitted to hospital within 30 days of discharge, and those who died after discharge. Univariate tests of difference between those admitted within 30 days or not, or living or deceased, were conducted using Fisher's exact test with categorical variables and the Wilcoxon rank sum test with continuous variables.

We conducted logistic regression of 30-day readmission and death after discharge, using purposeful selection of covariates [17]. Univariate logistic regression was conducted initially with all possible covariates. Any variable that contributed to the outcome at a significance level of at least $p=0.25$ was included in an initial multivariable model. This initial model was winnowed until only predictors achieving a significance level of $p<0.05$ remained in a model. All variables either initially excluded or dropped were tested again, one-by-one, in a model with only significant predictors. The linearity of all continuous covariates contributing significantly to the outcome was tested using fractional polynomial regression in STATA 15.1 (StataCorp, College Station, TX). Interactions between significant predictors that remained in a tentative final model were tested. A final model was generated with the Hosmer-Lemeshow test of goodness-of-fit, as well as a classification table and area under the receiver operating characteristic curve to evaluate sensitivity and specificity.

Finally, we generated a Kaplan-Meier estimator of the survival function, with a $95 \%$ confidence interval, of patients hospitalized with HRF $(n=202)$. Living participants were right-censored as of the last date of data collection (August $28,2018)$.

All analyses (other than fractional polynomial regression) were conducted using SAS 9.4 (SAS Institute, Cary, NC). Across all tests, statistical significance was defined as $p<0.05$ (2-tailed).

\section{Results}

Two hundred and two patients met eligibility criteria and were included in this study. Demographics are shown in Table 1. Fifteen patients died during the index admission (7\% of the population). Those who died were significantly older than those who survived to discharge ( 75.7 versus 62.4 years), and were significantly more likely to have an intensive care unit admission (93\% versus 56\%). There were no differences noted in co-morbidities or respiratory diagnoses of patients who died, and the Charlson comorbidity was similar, indicating similar chronic disease severity. There was no difference in arterial blood gas values or serum bicarbonate values between those who died during the index admission and those who survived until discharge. Those who died had significantly worse hemodynamics, measured by mean arterial blood pressure and need for pressor support, likely indicating increased acute severity of illness. 
Table 1 Demographics of hypercapnic respiratory failure participants by mortality status at index admission

\begin{tabular}{|c|c|c|c|c|}
\hline & All $(n=202)$ & $\begin{array}{l}\text { Living at dis- } \\
\text { charge }(n=187, \\
93 \%)\end{array}$ & Deceased $(n=15,7 \%)$ & $p$ \\
\hline Female & $113(56 \%)$ & $104(56 \%)$ & $9(60 \%)$ & 0.793 \\
\hline Age (years) & $63.4 \pm 15.3$ & $62.4 \pm 15.3$ & $75.7 \pm 9.9$ & $<0.001$ \\
\hline $\operatorname{BMI}\left(\mathrm{kg} / \mathrm{m}^{2}\right)$ & $36.4 \pm 14.2$ & $36.4 \pm 14.4$ & $36.5 \pm 11.9$ & 0.780 \\
\hline Active smoker & $63(31 \%)$ & $60(32 \%)$ & $3(20 \%)$ & 0.755 \\
\hline Length of admission (days) & $9.7 \pm 13.8$ & $9.9 \pm 14.2$ & $7.5 \pm 8.1$ & 0.775 \\
\hline \multicolumn{5}{|l|}{ Intensive care unit (ICU) admission } \\
\hline ICU admission & $119(59 \%)$ & $105(56 \%)$ & $14(93 \%)$ & 0.005 \\
\hline Intubation $^{\mathrm{a}}$ & $60(50 \%)$ & $51(49 \%)$ & $9(64 \%)$ & 0.394 \\
\hline \multicolumn{5}{|l|}{ Primary admission reason } \\
\hline Respiratory failure & $102(50 \%)$ & $93(50 \%)$ & $9(60 \%)$ & 0.593 \\
\hline Pneumonia & $22(11 \%)$ & $20(11 \%)$ & $2(13 \%)$ & 0.670 \\
\hline COPD exacerbation & $46(23 \%)$ & $43(23 \%)$ & $3(20 \%)$ & 1.000 \\
\hline Overdose & $10(5 \%)$ & $10(5 \%)$ & 0 & 1.000 \\
\hline Sepsis & $25(12 \%)$ & $24(13 \%)$ & $1(7 \%)$ & 0.699 \\
\hline Trauma & $6(3 \%)$ & $5(3 \%)$ & $1(7 \%)$ & 0.374 \\
\hline Cardiac disease & $58(29 \%)$ & $53(28 \%)$ & $5(33 \%)$ & 0.768 \\
\hline \multicolumn{5}{|l|}{ Comorbidities } \\
\hline Myocardial infarction & $26(13 \%)$ & $23(12 \%)$ & $3(20 \%)$ & 0.417 \\
\hline Congestive heart failure & $59(29 \%)$ & $54(29 \%)$ & $5(33 \%)$ & 0.770 \\
\hline Peripheral vascular disease & $14(7 \%)$ & $13(7 \%)$ & $1(7 \%)$ & 1.000 \\
\hline Cerebrovascular disease & $12(6 \%)$ & $10(5 \%)$ & $2(13 \%)$ & 0.220 \\
\hline Diabetes with chronic complications & $56(28 \%)$ & $52(28 \%)$ & $4(27 \%)$ & 1.000 \\
\hline Renal disease & $39(19 \%)$ & $36(19 \%)$ & $3(20 \%)$ & 1.000 \\
\hline \multicolumn{5}{|l|}{ Pulmonary disease } \\
\hline COPD & $93(46 \%)$ & $85(45 \%)$ & $8(53 \%)$ & 0.599 \\
\hline Obstructive sleep apnea & $49(24 \%)$ & $48(26 \%)$ & $1(7 \%)$ & 0.124 \\
\hline Obesity hypoventilation & $12(6 \%)$ & $12(6 \%)$ & 0 & 0.606 \\
\hline Asthma & $21(10 \%)$ & $21(11 \%)$ & 0 & 0.375 \\
\hline Interstitial lung disease & $6(3 \%)$ & $4(2 \%)$ & $2(13 \%)$ & 0.065 \\
\hline Bronchiectasis (including CF) & $3(1 \%)$ & $3(2 \%)$ & 0 & 1.000 \\
\hline Obstructive sleep apnea & $49(24 \%)$ & $48(26 \%)$ & $1(7 \%)$ & 0.124 \\
\hline \multicolumn{5}{|c|}{ Prior to admission home respiratory support } \\
\hline CPAP & $24(12 \%)$ & $23(12 \%)$ & $1(7 \%)$ & 1.000 \\
\hline BIPAP & $17(8 \%)$ & $16(9 \%)$ & $1(7 \%)$ & 1.000 \\
\hline Oxygen & $75(37 \%)$ & $69(37 \%)$ & $6(40 \%)$ & 0.789 \\
\hline None & $107(53 \%)$ & $100(53 \%)$ & $7(47 \%)$ & 0.789 \\
\hline Charlson comorbidity index ${ }^{b}$ & $2.0 \pm 1.6$ & $2.0 \pm 1.6$ & $1.8 \pm 1.8$ & 0.478 \\
\hline \multicolumn{5}{|c|}{ Admission or death due to hypercapnic respiratory failure } \\
\hline Yes & $129(64 \%)$ & $118(63 \%)$ & $11(73 \%)$ & 0.922 \\
\hline No & $41(20 \%)$ & $39(21 \%)$ & $2(13 \%)$ & \\
\hline Maybe & $30(15 \%)$ & $28(15 \%)$ & $2(13 \%)$ & \\
\hline \multicolumn{5}{|l|}{ Arterial blood gas values ${ }^{c}$} \\
\hline $\begin{array}{l}\mathrm{Pa}_{\mathrm{CO}_{2}} \\
\text { Serum bicarbonate }\end{array}$ & $60.6 \pm 24.3$ & $60.3 \pm 23.4$ & $64.2 \pm 32.4$ & 0.714 \\
\hline$<15$ & $1(0.5 \%)$ & $1(0.5 \%)$ & 0 & 0.709 \\
\hline $15-19$ & $9(4 \%)$ & $8(4 \%)$ & $1(7 \%)$ & \\
\hline $20-29$ & $97(48 \%)$ & $88(47 \%)$ & $9(60 \%)$ & \\
\hline $30-39$ & $75(37 \%)$ & $70(37 \%)$ & $5(33 \%)$ & \\
\hline $40-49$ & $11(5 \%)$ & $11(6 \%)$ & 0 & \\
\hline
\end{tabular}


Table 1 (continued)

\begin{tabular}{|c|c|c|c|c|}
\hline & All $(n=202)$ & $\begin{array}{l}\text { Living at dis- } \\
\text { charge }(n=187, \\
93 \%)\end{array}$ & Deceased $(n=15,7 \%)$ & $p$ \\
\hline \multicolumn{5}{|l|}{ Hemodynamics in first $24 \mathrm{~h}$} \\
\hline \multicolumn{5}{|l|}{ Most extreme heart rate } \\
\hline$<40$ & $1(0.5 \%)$ & $1(0.5 \%)$ & 0 & \multirow[t]{5}{*}{0.134} \\
\hline $40-69$ & $26(13 \%)$ & $21(11 \%)$ & $5(33 \%)$ & \\
\hline $70-119$ & $135(67 \%)$ & $128(68 \%)$ & $7(47 \%)$ & \\
\hline $120-159$ & $31(15 \%)$ & $28(15 \%)$ & $3(20 \%)$ & \\
\hline$\geq 160$ & $3(1 \%)$ & $3(2 \%)$ & 0 & \\
\hline \multicolumn{5}{|l|}{ Lowest blood pressure } \\
\hline $\mathrm{MAP} \geq 70$ & $148(73 \%)$ & $142(76 \%)$ & $6(40 \%)$ & \multirow[t]{4}{*}{$<0.001$} \\
\hline $\mathrm{MAP}<70$ & $35(17 \%)$ & $33(18 \%)$ & $2(13 \%)$ & \\
\hline Low dose vasopressor ${ }^{\mathrm{d}}$ & $6(3 \%)$ & $4(2 \%)$ & $2(13 \%)$ & \\
\hline High dose vasopressor ${ }^{\mathrm{d}}$ & $11(5 \%)$ & $6(3 \%)$ & $5(33 \%)$ & \\
\hline
\end{tabular}

Bold values indicate statistical significance

Values shown are mean and SD, and number (\%). Categorical variables were tested using Fisher's exact test. Continuous variables were tested using the Wilcoxon rank sum test

${ }^{a}$ Among those admitted to the ICU $(n=119)$

${ }^{\mathrm{b}}$ Based on Quan et al. [15]

c37-39\% Data missing

${ }^{\mathrm{d}}$ Low dose defined as dopamine $\leq 15$ or epinephrine $\leq 0.1$ or norepinephrine $\leq 0.1 \mathrm{mcg} / \mathrm{kg} / \mathrm{min}$, and high dose defined as dopamine $>15$ or epinephrine $>0.1$ or norepinephrine $>0.1 \mathrm{mcg} / \mathrm{kg} / \mathrm{min}$

Overall, patients had a long period of hospitalization; the average hospital length of stay was nearly 10 days in those surviving to discharge, and 7.5 in those who died during the admission.

We next investigated 30-day readmission for those who survived to hospital discharge; demographics are shown in Table 2, logistic regression analysis in Table 3. There was a high rate of readmission within 30 days: $23 \%$ of patients were readmitted during this time. Among those readmitted within 30 days fewer were smokers (17\% versus $36 \%$ ), and more had peripheral vascular disease (17\% versus $4 \%$ ), interstitial lung disease ( $7 \%$ versus $1 \%$ ), bronchiectasis ( $7 \%$ versus $0 \%$ ) and tachycardia during the first $24 \mathrm{~h}$. Multi-variate logistic regression showed only a diagnosis of peripheral vascular disease [adjusted odds ratio (aOR) 4.78, CI 1.45-15.74] and a rapid heart rate in the first $24 \mathrm{~h}$ (aOR 2.97, CI 1.22, 7.26) were associated with increased risk of readmission.

We investigated characteristics of participants who died following discharge, compared to those who survived (demographics shown in Table 4, logistic regression analysis in $3 b$ ). Those who died were significantly older (69.0 versus 58.6 years), fewer were active smokers (19\% versus $39 \%$ ) or had been intubated during the index admission ( $26 \%$ versus $60 \%$ ). Patients who died were more often admitted with a primary diagnosis related to cardiac disease $(41 \%$ versus $21 \%$ ), have comorbidities of congestive heart failure (44\% versus $20 \%$ ), peripheral vascular disease (16\% versus $2 \%$ ), malignancy ( $15 \%$ versus $5 \%$ ), and COPD (56\% versus $39 \%$ ).
More who died were on home oxygen (53\% versus 33\%) and readmitted within 30 days ( $34 \%$ versus $15 \%$ ). Those who died following discharge had a lower $\mathrm{Pa}_{\mathrm{O}_{2}}$ (but also were on a lower level of supplemental oxygen) and higher serum bicarbonate during the index admission.

Multi-variable logistic regression showed the following factors predicted risk of death (Table 5): for every 5 years increased age, the aOR of death was $1.32(1.13$, 1.54); the adjusted odds of death in those using home oxygen at the time of the index admission was 4.03 (1.89, 8.57); the adjusted odds of death in those with peripheral vascular disease was 12.56 (CI 2.35-67.21); for every unit increase in the Quan-Charlson comorbidity index, the risk of death increased by 1.39 (CI 1.09-1.76); and the adjusted odds of death in those readmitted to hospital was 3.07 (CI 1.46-6.43).

Timing of death relative to the index admission is shown in Fig. 1, notably mortality is particularly high during the first few months after hospital discharge.

\section{Discussion}

Patients admitted to an academic medical center with a diagnosis of HRF were assessed regarding outcomes during their index admission, risk of 30-day readmission and risk of death. Our results suggest that patients admitted with a diagnosis of HRF have significant mortality during 
Table 2 Demographics of hypercapnic respiratory failure participants by 30-day readmission status

\begin{tabular}{|c|c|c|c|c|}
\hline & All $(n=181)$ & $\begin{array}{l}\text { Not readmitted within } 30 \text { days } \\
(n=140,77 \%)\end{array}$ & $\begin{array}{l}\text { Readmitted within } 30 \text { days } \\
(n=41,23 \%)\end{array}$ & $p$ \\
\hline Female & $100(55 \%)$ & $78(56 \%)$ & $22(54 \%)$ & 0.859 \\
\hline Age (years) & $62.3 \pm 15.3$ & $62.3 \pm 14.2$ & $62.5 \pm 18.8$ & 0.451 \\
\hline BMI $\left(\mathrm{kg} / \mathrm{m}^{2}\right)$ & $36.6 \pm 14.4$ & $37.4 \pm 15.2$ & $33.7 \pm 10.8$ & 0.250 \\
\hline Active smoker & $58(32 \%)$ & $51(36 \%)$ & $7(17 \%)$ & 0.022 \\
\hline Length of admission $(M \pm \mathrm{SD})$ (days) & $9.6 \pm 13.9$ & $9.6 \pm 14.1$ & $9.8 \pm 13.4$ & 0.766 \\
\hline \multicolumn{5}{|l|}{ ICU admission } \\
\hline ICU admission & $103(57 \%)$ & $84(60 \%)$ & $19(46 \%)$ & 0.151 \\
\hline More than one ICU admission ${ }^{\mathrm{a}}$ & $15(15 \%)$ & $10(12 \%)$ & $5(26 \%)$ & 0.146 \\
\hline Intubation $^{\mathrm{a}}$ & $49(48 \%)$ & $43(51 \%)$ & $6(32 \%)$ & 0.136 \\
\hline \multicolumn{5}{|l|}{ Primary admission reason } \\
\hline Respiratory failure & $90(50 \%)$ & $74(53 \%)$ & $16(39 \%)$ & 0.155 \\
\hline Pneumonia & $18(10 \%)$ & $15(11 \%)$ & $3(7 \%)$ & 0.767 \\
\hline COPD exacerbation & $43(24 \%)$ & $35(25 \%)$ & $8(20 \%)$ & 0.537 \\
\hline Overdose & $10(5 \%)$ & $10(7 \%)$ & 0 & 0.120 \\
\hline Sepsis & $23(13 \%)$ & $20(14 \%)$ & $3(7 \%)$ & 0.296 \\
\hline Trauma & $3(2 \%)$ & $2(1 \%)$ & $1(2 \%)$ & 0.539 \\
\hline Cardiac disease & $52(29 \%)$ & $36(26 \%)$ & $16(39 \%)$ & 0.117 \\
\hline \multicolumn{5}{|l|}{ Comorbidities } \\
\hline Myocardial infarction & $23(13 \%)$ & $17(12 \%)$ & $6(15 \%)$ & 0.790 \\
\hline Congestive heart failure & $54(30 \%)$ & $41(29 \%)$ & $13(32 \%)$ & 0.846 \\
\hline Peripheral vascular disease & $13(7 \%)$ & $6(4 \%)$ & $7(17 \%)$ & 0.011 \\
\hline Cerebrovascular disease & $10(5 \%)$ & $8(6 \%)$ & $2(5 \%)$ & 1.000 \\
\hline Dementia & $1(1 \%)$ & $1(1 \%)$ & 0 & 1.000 \\
\hline Diabetes without chronic complications & $28(15 \%)$ & $19(14 \%)$ & $9(22 \%)$ & 0.221 \\
\hline Renal disease & $35(19 \%)$ & $27(19 \%)$ & $8(20 \%)$ & 1.000 \\
\hline \multicolumn{5}{|l|}{ Pulmonary disease } \\
\hline COPD & $85(47 \%)$ & $71(51 \%)$ & $14(34 \%)$ & 0.076 \\
\hline Obstructive sleep apnea & $45(25 \%)$ & $36(26 \%)$ & $9(22 \%)$ & 0.686 \\
\hline Obesity hypoventilation & $12(7 \%)$ & $11(8 \%)$ & $1(2 \%)$ & 0.303 \\
\hline Asthma & $21(12 \%)$ & $17(12 \%)$ & $4(10 \%)$ & 0.788 \\
\hline Interstitial lung disease & $4(2 \%)$ & $1(1 \%)$ & $3(7 \%)$ & 0.037 \\
\hline Bronchiectasis (including CF) & $3(2 \%)$ & 0 & $3(7 \%)$ & 0.011 \\
\hline \multicolumn{5}{|l|}{ Home respiratory support } \\
\hline CPAP & $22(12 \%)$ & $17(12 \%)$ & $5(12 \%)$ & 1.000 \\
\hline BIPAP & $16(9 \%)$ & $13(9 \%)$ & $3(7 \%)$ & 1.000 \\
\hline Oxygen & $69(38 \%)$ & $55(39 \%)$ & $14(34 \%)$ & 0.588 \\
\hline None & $95(52 \%)$ & $70(50 \%)$ & $25(61 \%)$ & 0.286 \\
\hline Charlson comorbidity index ${ }^{b}$ & $2.0 \pm 1.6$ & $2.1 \pm 1.6$ & $1.8 \pm 1.5$ & 0.224 \\
\hline \multicolumn{5}{|l|}{ Discharge respiratory support } \\
\hline CPAP & $12(7 \%)$ & $8(6 \%)$ & $4(10 \%)$ & 0.473 \\
\hline BIPAP & $22(12 \%)$ & $19(14 \%)$ & $3(7 \%)$ & 0.416 \\
\hline Oxygen & $73(40 \%)$ & $57(41 \%)$ & $16(39 \%)$ & 1.000 \\
\hline None & $86(47 \%)$ & $65(46 \%)$ & $21(51 \%)$ & 0.599 \\
\hline \multicolumn{5}{|c|}{ Admission or death due to hypercapnic respiratory failure } \\
\hline Yes & $115(63 \%)$ & $90(64 \%)$ & $25(61 \%)$ & 0.756 \\
\hline No & $37(20 \%)$ & $27(19 \%)$ & $10(24 \%)$ & \\
\hline Maybe & $27(15 \%)$ & $21(15 \%)$ & $6(15 \%)$ & \\
\hline \multicolumn{5}{|c|}{ Readmission due to hypercapnic respiratory failure } \\
\hline Yes & $66(36 \%)$ & $39(28 \%)$ & $27(66 \%)$ & $<0.001$ \\
\hline
\end{tabular}


Table 2 (continued)

\begin{tabular}{|c|c|c|c|c|}
\hline & All $(n=181)$ & $\begin{array}{l}\text { Not readmitted within } 30 \text { days } \\
(n=140,77 \%)\end{array}$ & $\begin{array}{l}\text { Readmitted within } 30 \text { days } \\
(n=41,23 \%)\end{array}$ & $p$ \\
\hline No & $31(17 \%)$ & $21(15 \%)$ & $10(24 \%)$ & \\
\hline Maybe & $9(5 \%)$ & $5(4 \%)$ & $4(10 \%)$ & \\
\hline Deceased during study period & $68(38 \%)$ & $45(32 \%)$ & $23(56 \%)$ & 0.010 \\
\hline \multicolumn{5}{|l|}{ Arterial blood gas values ${ }^{c}$} \\
\hline $\mathrm{Pa}_{\mathrm{CO}_{2}}$ & $59.9 \pm 22.9$ & $60.8 \pm 24.2$ & $55.5 \pm 15.1$ & 0.570 \\
\hline \multicolumn{5}{|l|}{ Bicarbonate } \\
\hline$<15$ & $1(1 \%)$ & $1(1 \%)$ & 0 & 0.289 \\
\hline $15-19$ & $8(4 \%)$ & $5(4 \%)$ & $3(7 \%)$ & \\
\hline $20-29$ & $84(46 \%)$ & $65(46 \%)$ & $19(46 \%)$ & \\
\hline $30-39$ & $69(38 \%)$ & $51(36 \%)$ & $18(44 \%)$ & \\
\hline $40-49$ & $10(6 \%)$ & $10(7 \%)$ & 0 & \\
\hline \multicolumn{5}{|l|}{ Hemodynamics in first $24 \mathrm{~h}$} \\
\hline \multicolumn{5}{|c|}{ Most extreme heart rate in first $24 \mathrm{~h}$} \\
\hline$<40$ & $1(1 \%)$ & 0 & $1(2 \%)$ & 0.017 \\
\hline $40-69$ & $21(12 \%)$ & $14(10 \%)$ & $7(17 \%)$ & \\
\hline $70-119$ & $123(68 \%)$ & $101(72 \%)$ & $22(54 \%)$ & \\
\hline $120-159$ & $27(15 \%)$ & $16(11 \%)$ & $11(27 \%)$ & \\
\hline$\geq 160$ & $3(2 \%)$ & $3(2 \%)$ & 0 & \\
\hline \multicolumn{5}{|l|}{ Lowest blood pressure } \\
\hline $\mathrm{MAP} \geq 70$ & $137(76 \%)$ & $105(75 \%)$ & $32(78 \%)$ & 1.000 \\
\hline $\mathrm{MAP}<70$ & $32(18 \%)$ & $25(18 \%)$ & $7(17 \%)$ & \\
\hline Low dose vasopressor ${ }^{\mathrm{d}}$ & $4(2 \%)$ & $3(2 \%)$ & $1(2 \%)$ & \\
\hline High dose vasopressor ${ }^{\mathrm{d}}$ & $6(3 \%)$ & $5(4 \%)$ & $1(2 \%)$ & \\
\hline
\end{tabular}

Bold values indicate statistical significance

Values shown are mean and SD, and number (\%). Categorical variables were tested using Fisher's exact test. Continuous variables were tested using the Wilcoxon rank sum test

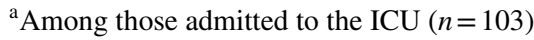

${ }^{\mathrm{b}}$ Based on Quan et al. [15]

${ }^{\mathrm{c}} 38-41 \%$ Data missing

${ }^{\mathrm{d}}$ Low dose defined as dopamine $\leq 15$ or epinephrine $\leq 0.1$ or norepinephrine $\leq 0.1 \mathrm{mcg} / \mathrm{kg} / \mathrm{min}$, and high dose defined as dopamine $>15$ or epinephrine $>0.1$ or norepinephrine $>0.1 \mathrm{mcg} / \mathrm{kg} / \mathrm{min}$

their index admission, a high 30-day readmission rate, and a high mortality rate overall. Certain characteristics independently identify patients at particularly high risk of adverse outcomes: older age, a diagnosis of peripheral vascular disease, and use of home oxygen.

Patients with HRF have a high mortality rate during their index admission - in the current study, 7\% died during their index admission. There are few prior studies reporting on the inpatient mortality of patients with HRF, as most prior publications have focused exclusively on patients admitted with acute HRF requiring NIV. Ahmed reported that 53\% of patients requiring NIV died during hospitalization and Lemyze found a $30 \%$ mortality rate among obese patients treated with NIV for acute respiratory failure [18]. Our study differs from these prior studies, which focused only on patients requiring NIV, which likely explains the lower initial mortality rate observed in our study.
Patients with a diagnosis of HRF had a very high 30-day readmission rate-23\%. Of those patients readmitted within 30 days, $66 \%$ were readmitted with a diagnosis of HRF. While these patients may have been stable enough for initial discharge, these data suggest that a diagnosis of HRF identifies a patient population at high risk of 30-day readmission. The 30-day readmission rates found in this population are significantly higher than those found for other conditions previously recognized to have a high readmission rate, such as acute myocardial infarction (14.8\%), COPD exacerbation $(7-20 \%)[19,20]$ or pneumonia $(16.7 \%)$ [21, 22]. HRF appears to be a marker of patients at particularly high risk of early hospital readmission (Table 5).

Certain co-morbidities were associated with an increased risk of 30-day readmission. Active smokers were less likely to be readmitted, but this was not significant in multi-variate regression, suggesting that still smoking was related to some 
Table 3 Unadjusted and adjusted odds ratios (and 95\% confidence intervals) from logistic regression analyses predicting 30-day readmission

\begin{tabular}{|c|c|c|c|c|}
\hline & Unadjusted OR & $95 \% \mathrm{CI}$ & Adjusted OR & $95 \% \mathrm{CI}$ \\
\hline Sex: Male vs. female & 1.087 & $(0.540,2.185)$ & & \\
\hline Age (by 5-year units) & 1.003 & $(0.895,1.124)$ & & \\
\hline BMI & 0.979 & $(0.952,1.007)$ & & \\
\hline Active smoker: (yes vs. no) & 0.347 & $(0.143,0.841)$ & & \\
\hline Length of admission (days) & 1.001 & $(0.977,1.026)$ & & \\
\hline \multicolumn{5}{|l|}{ Intensive care unit admission } \\
\hline (Yes vs. no) & 0.576 & $(0.286,1.160)$ & & \\
\hline Intubation: (yes vs. no) ${ }^{\mathrm{a}}$ & 0.387 & $(0.151,0.988)$ & & \\
\hline \multicolumn{5}{|l|}{ Primary admission reason } \\
\hline Respiratory failure (yes vs. no) & 0.571 & $(0.281,1.161)$ & & \\
\hline Pneumonia (yes vs. no) & 0.658 & $(0.181,2.395)$ & & \\
\hline COPD exacerbation (yes vs. no) & 0.727 & $(0.307,1.722)$ & & \\
\hline Overdose (yes vs. no) & $<0.001$ & $(<0.001,>999)$ & & \\
\hline Sepsis (yes vs. no) & 0.474 & $(0.133,1.682)$ & & \\
\hline Trauma (yes vs. no) & 1.726 & $(0.153,19.528)$ & & \\
\hline Cardiac disease (yes vs. no) & 1.849 & $(0.888,3.848)$ & & \\
\hline Myocardial infarction (yes vs. no) & 1.240 & $(0.455,3.384)$ & & \\
\hline Congestive heart failure (yes vs. no) & 1.121 & $(0.529,2.378)$ & & \\
\hline Peripheral vascular disease (yes vs. no) & 4.598 & $(1.451,14.573)$ & 4.779 & $(1.451,15.744)$ \\
\hline Cerebrovascular disease (yes vs. no) & 0.846 & $(0.173,4.150)$ & & \\
\hline Chronic pulmonary disease (yes vs. no) & 0.646 & $(0.318,1.313)$ & & \\
\hline Diabetes with chronic complications (yes vs. no) & 0.806 & $(0.362,1.798)$ & & \\
\hline Renal disease (yes vs. no) & 1.015 & $(0.421,2.444)$ & & \\
\hline Obstructive sleep apnea (yes vs. no) & 0.813 & $(0.354,1.865)$ & & \\
\hline \multicolumn{5}{|l|}{ Pulmonary disease } \\
\hline COPD (yes vs. no) & 0.504 & $(0.244,1.041)$ & & \\
\hline Obesity hypoventilation (yes vs. no) & 0.293 & $(0.037,2.341)$ & & \\
\hline Asthma (yes vs. no) & 0.782 & $(0.248,2.469)$ & & \\
\hline Interstitial lung disease (yes vs. no) & 10.974 & $(1.110,108.519)$ & & \\
\hline Bronchiectasis (including CF) (yes vs. no) & $>999$ & $(<0.001,>999)$ & & \\
\hline \multicolumn{5}{|l|}{ Home respiratory support } \\
\hline CPAP (yes vs. no) & 1.005 & $(0.347,2.912)$ & & \\
\hline BIPAP (yes vs. no) & 0.771 & $(0.209,2.849)$ & & \\
\hline Oxygen & 0.801 & $(0.386,1.662)$ & & \\
\hline \multicolumn{5}{|l|}{ None } \\
\hline Charlson comorbidity index ${ }^{\mathrm{b}}$ & 0.863 & $(0.683,1.090)$ & & \\
\hline \multicolumn{5}{|l|}{ Discharge respiratory support } \\
\hline CPAP (yes vs. no) & 1.784 & $(0.509,6.253)$ & & \\
\hline BIPAP (yes vs. no) & 0.503 & $(0.141,1.792)$ & & \\
\hline Oxygen (yes vs. no) & 0.932 & $(0.457,1.900)$ & & \\
\hline None (yes vs. no) & 1.212 & $(0.604,2.432)$ & & \\
\hline \multicolumn{5}{|c|}{ Admission or death due to hypercapnic respiratory failure } \\
\hline Yes vs. no & 0.750 & $(0.321,1.755)$ & & \\
\hline Maybe vs. no & 0.771 & $(0.241,2.465)$ & & \\
\hline \multicolumn{5}{|l|}{ Readmission } \\
\hline Yes vs. no & $>999$ & $(<0.001,>999)$ & & \\
\hline Days until first readmission ${ }^{c}$ & 0.603 & $(0.368,0.989)$ & & \\
\hline Number of readmissions ${ }^{c}$ & 1.111 & $(0.904,1.366)$ & & \\
\hline \multicolumn{5}{|l|}{ Readmission due to hypercapnic respiratory failure } \\
\hline Yes vs. no & 1.454 & $(0.592,3.572)$ & & \\
\hline
\end{tabular}


Table 3 (continued)

\begin{tabular}{|c|c|c|c|c|}
\hline & Unadjusted OR & $95 \% \mathrm{CI}$ & Adjusted OR & $95 \% \mathrm{CI}$ \\
\hline Maybe vs. no & 1.680 & $(0.369,7.644)$ & & \\
\hline N/A vs. no & $<0.001$ & $(<0.001,>999)$ & & \\
\hline \multicolumn{5}{|l|}{ Arterial blood gas values ${ }^{\mathrm{d}}$} \\
\hline $\mathrm{Pa}_{\mathrm{CO}_{2}}$ & 0.989 & $(0.966,1.013)$ & & \\
\hline \multicolumn{5}{|l|}{ Bicarbonate } \\
\hline$\leq 19$ vs. $20-29$ & 1.711 & $(0.390,7.493)$ & & \\
\hline $30-39$ vs. $20-29$ & 1.207 & $(0.575,2.535)$ & & \\
\hline $40-49$ vs. $20-29$ & $<0.001$ & $(0.001,>999)$ & & \\
\hline \multicolumn{5}{|c|}{ Hemodynamics in first $24 \mathrm{~h}$} \\
\hline \multicolumn{5}{|c|}{ Most extreme heart rate in first $24 \mathrm{~h}$} \\
\hline$\leq 69$ vs. $70-119$ & 2.623 & $(0.981,7.014)$ & 2.464 & $(0.891,6.814)$ \\
\hline$\geq 120$ vs. $70-119$ & 2.658 & $(1.109,6.370)$ & 2.970 & $(1.215,7.261)$ \\
\hline \multicolumn{5}{|l|}{ Lowest blood pressure } \\
\hline Other vs. MAP $\geq 70$ & 0.895 & $(0.388,2.066)$ & & \\
\hline
\end{tabular}

Bold values indicate statistical significance

$O R$ odds ratio, $C I$ confidence interval

${ }^{a}$ Among those admitted to the ICU $(n=103)$

${ }^{\mathrm{b}}$ Based on Quan et al. [15]

${ }^{\mathrm{c}}$ Among those readmitted $(n=95)$

${ }^{\mathrm{d}} 38-41 \%$ Data missing

other factor such as perhaps reduced overall level of chronic illness, rather than an independent factor. A diagnosis of peripheral vascular disease identified patients at particularly high risk of readmission in multivariate regression analysis. Peripheral vascular disease has been identified as a risk factor for readmission following catheter ablation for atrial fibrillation [23], percutaneous coronary intervention [24], admission for heart failure [25] and acute hospitalizations in older adults [26], suggesting that peripheral vascular disease may particularly be a marker of chronic illness severity that increases risk of readmission for patients. Another factor that predicted an increased risk of 30-day readmission in this cohort was tachycardia during the first $24 \mathrm{~h}$ of the index hospitalization. Tachycardia would appear to be a factor limited to acute illness during the index admission. We are not able to explain why this would predicted subsequent 30-day readmission, but perhaps it is a marker of chronic cardiovascular dysfunction, which might predict increased risk. Identifying these patients as being at high risk of 30-day readmission may be a first step to providing targeted interventions to prevent readmission.

Only $17 \%$ of patients were discharged on any form of ventilatory support [and this included only continuous positive airway pressure (CPAP) or bilevel positive airway pressure (BIPAP)]. Use of CPAP or BIPAP did not appear to affect outcomes, but as there were so few participants discharged on these modalities, it is somewhat difficult to interpret these data. Thirty two percent of patients admitted with HRF had comorbid OSA or obesity hypoventilation, indications for ventilatory support, and so there are likely many patients who could have benefited from institution of CPAP, BIPAP or NIV on discharge for these indications. There are also likely to be patients with chronic pulmonary disease that might benefit from ventilatory support: Murphy et al. reported that NIV for patients with persistent hypercapnia and hypoxemia 2-4 weeks following hospital discharge significantly reduced the risk of readmission or death at 12 months [27], and Kohnlein et al. reported that an NIV intervention in patients with chronic hypercapnia targeting reduction in $\mathrm{Pa}_{\mathrm{CO}_{2}}$ significantly reduced 12 month mortality [28]. Some patients with COPD likely have the overlap syndrome (overlap of COPD with OSA), and an observational study by Marin et al. suggested that treatment of such patients with CPAP reduces the risk of death and COPD exacerbation [2]. The data supporting NIV for patients with COPD suggests this intervention is most effective when initiated after the acute exacerbation, so it is possible that many patients were not discharged on CPAP or BIPAP as it was not deemed medically necessary, though such patients should be closely followed up on discharge. Our study suggests that there may be an opportunity to increase the rate of ventilatory support for some patients.

Thirty-six patients died in the period following hospital discharge (follow-up period 19-31 months). A number of co-morbidities and factors were more common in those who died, but factors that were independent risk factors for death 
Table 4 Demographics of hypercapnic respiratory failure participants by mortality status after discharge

\begin{tabular}{|c|c|c|c|c|}
\hline & All $(n=187)$ & $\begin{array}{l}\text { Living after dis- } \\
\text { charge }(n=119)\end{array}$ & $\begin{array}{l}\text { Deceased after } \\
\text { discharge }(n=68)\end{array}$ & $p$ \\
\hline Female & $104(56 \%)$ & $65(55 \%)$ & $39(57 \%)$ & 0.761 \\
\hline Age (years) & $62.4 \pm 15.3$ & $58.6 \pm 15.9$ & $69.0 \pm 11.6$ & $<0.001$ \\
\hline BMI & $36.4 \pm 14.4$ & $37.3 \pm 15.0$ & $34.8 \pm 13.2$ & 0.196 \\
\hline Active smoker & $60(32 \%)$ & $47(39 \%)$ & $13(19 \%)$ & 0.005 \\
\hline Length of admission (days) & $9.9 \pm 14.2$ & $11.8 \pm 16.8$ & $6.7 \pm 6.4$ & 0.089 \\
\hline \multicolumn{5}{|l|}{ Intensive care unit admission } \\
\hline ICU admission & $105(56 \%)$ & $70(59 \%)$ & $35(51 \%)$ & 0.360 \\
\hline Length of ICU admission (days) ${ }^{a}$ & $8.1 \pm 35.4$ & $5.5 \pm 7.6$ & $13.4 \pm 60.6$ & 0.266 \\
\hline More than one ICU admission ${ }^{\mathrm{a}}$ & $15(14 \%)$ & $8(11 \%)$ & $7(20 \%)$ & 0.250 \\
\hline Intubation $^{\mathrm{a}}$ & $51(49 \%)$ & $42(60 \%)$ & $9(26 \%)$ & 0.001 \\
\hline \multicolumn{5}{|l|}{ Primary admission reason } \\
\hline Respiratory failure & $93(50 \%)$ & $55(46 \%)$ & $38(56 \%)$ & 0.226 \\
\hline Pneumonia & $20(11 \%)$ & $12(10 \%)$ & $8(12 \%)$ & 0.807 \\
\hline COPD exacerbation & $43(23 \%)$ & $22(18 \%)$ & $21(31 \%)$ & 0.070 \\
\hline Overdose & $10(5 \%)$ & $8(7 \%)$ & $2(3 \%)$ & 0.332 \\
\hline Sepsis & $24(13 \%)$ & $19(16 \%)$ & $5(7 \%)$ & 0.113 \\
\hline Trauma & $5(3 \%)$ & $4(3 \%)$ & $1(1 \%)$ & 0.654 \\
\hline Cardiac disease & $53(28 \%)$ & $25(21 \%)$ & $28(41 \%)$ & 0.004 \\
\hline \multicolumn{5}{|l|}{ Comorbidities } \\
\hline Myocardial infarction & $23(12 \%)$ & $12(10 \%)$ & $11(16 \%)$ & 0.251 \\
\hline Congestive heart failure & $54(29 \%)$ & $24(20 \%)$ & $30(44 \%)$ & 0.001 \\
\hline Peripheral vascular disease & $13(7 \%)$ & $2(2 \%)$ & $11(16 \%)$ & $<0.001$ \\
\hline Cerebrovascular disease & $10(5 \%)$ & $4(3 \%)$ & $6(9 \%)$ & 0.173 \\
\hline Diabetes with chronic complications & $52(28 \%)$ & $36(30 \%)$ & $16(24 \%)$ & 0.397 \\
\hline Renal disease & $36(19 \%)$ & $18(15 \%)$ & $18(26 \%)$ & 0.082 \\
\hline \multicolumn{5}{|l|}{ Pulmonary disease } \\
\hline COPD & $85(45 \%)$ & $47(39 \%)$ & $38(56 \%)$ & 0.034 \\
\hline Obstructive sleep apnea & $48(26 \%)$ & $33(28 \%)$ & $15(22 \%)$ & 0.487 \\
\hline Obesity hypoventilation & $12(6 \%)$ & $6(5 \%)$ & $6(9 \%)$ & 0.359 \\
\hline Asthma & $21(11 \%)$ & $15(13 \%)$ & $6(9 \%)$ & 0.481 \\
\hline Interstitial lung disease & $4(2 \%)$ & $1(1 \%)$ & $3(4 \%)$ & 0.137 \\
\hline Bronchiectasis (including CF) & $3(2 \%)$ & $3(3 \%)$ & 0 & 0.555 \\
\hline \multicolumn{5}{|c|}{ Prior to admission home respiratory support } \\
\hline CPAP & $23(12 \%)$ & $17(14 \%)$ & $6(9 \%)$ & 0.357 \\
\hline BIPAP & $16(9 \%)$ & $8(7 \%)$ & $8(12 \%)$ & 0.280 \\
\hline Oxygen & $69(37 \%)$ & $33(28 \%)$ & $36(53 \%)$ & 0.001 \\
\hline None & $100(53 \%)$ & $71(60 \%)$ & $29(43 \%)$ & $\mathbf{0 . 0 3 3}$ \\
\hline Charlson comorbidity index ${ }^{b}$ & $2.0 \pm 1.6$ & $1.6 \pm 1.5$ & $2.6 \pm 1.6$ & $<0.001$ \\
\hline \multicolumn{5}{|l|}{ Discharge respiratory support } \\
\hline CPAP & $12(6 \%)$ & $7(6 \%)$ & $5(7 \%)$ & 0.760 \\
\hline BIPAP & $22(12 \%)$ & $12(10 \%)$ & $10(15 \%)$ & 0.355 \\
\hline Oxygen & $75(40 \%)$ & $39(33 \%)$ & $36(53 \%)$ & 0.008 \\
\hline Other non-invasive ventilation & 0 & 0 & 0 & - \\
\hline None & $91(49 \%)$ & $67(56 \%)$ & $24(35 \%)$ & 0.006 \\
\hline \multicolumn{5}{|c|}{ Admission or death due to hypercapnic respiratory failure } \\
\hline Yes & $118(63 \%)$ & $73(61 \%)$ & $45(66 \%)$ & 0.754 \\
\hline No & $39(21 \%)$ & $27(23 \%)$ & $12(18 \%)$ & \\
\hline Maybe & $28(15 \%)$ & $18(15 \%)$ & $10(15 \%)$ & \\
\hline \multicolumn{5}{|l|}{ Readmissions } \\
\hline Readmission & $98(52 \%)$ & $50(42 \%)$ & $48(71 \%)$ & $<0.001$ \\
\hline
\end{tabular}


Table 4 (continued)

\begin{tabular}{|c|c|c|c|c|}
\hline & All $(n=187)$ & $\begin{array}{l}\text { Living after dis- } \\
\text { charge }(n=119)\end{array}$ & $\begin{array}{l}\text { Deceased after } \\
\text { discharge }(n=68)\end{array}$ & $p$ \\
\hline Readmission within 30 days $^{\mathrm{c}}$ & $41(22 \%)$ & $18(15 \%)$ & $23(34 \%)$ & 0.005 \\
\hline Number of readmissions & $2.6 \pm 2.0$ & $2.7 \pm 2.0$ & $2.4 \pm 2.0$ & 0.250 \\
\hline \multicolumn{5}{|c|}{ Readmission due to hypercapnic respiratory failure } \\
\hline Yes & $68(62 \%)$ & $31(50 \%)$ & $37(77 \%)$ & \multirow[t]{3}{*}{0.015} \\
\hline No & $32(29 \%)$ & $23(37 \%)$ & $9(19 \%)$ & \\
\hline Maybe & $10(9 \%)$ & $8(13 \%)$ & $2(4 \%)$ & \\
\hline \multicolumn{5}{|l|}{ Arterial blood gas values ${ }^{\mathrm{d}}$} \\
\hline \multicolumn{5}{|l|}{ Bicarbonate } \\
\hline$<15$ & $1(0.5 \%)$ & 0 & $1(1 \%)$ & \multirow[t]{5}{*}{0.002} \\
\hline $15-19$ & $8(4 \%)$ & $6(5 \%)$ & $2(3 \%)$ & \\
\hline $20-29$ & $88(47 \%)$ & $65(55 \%)$ & $23(34 \%)$ & \\
\hline $30-39$ & $70(37 \%)$ & $37(31 \%)$ & $33(49 \%)$ & \\
\hline $40-49$ & $11(6 \%)$ & $3(3 \%)$ & $8(12 \%)$ & \\
\hline \multicolumn{5}{|l|}{ Hemodynamics in first $24 \mathrm{~h}$} \\
\hline \multicolumn{5}{|c|}{ Most extreme heart rate in first $24 \mathrm{~h}$} \\
\hline$<40$ & $1(0.5 \%)$ & 0 & $1(1 \%)$ & \multirow[t]{5}{*}{0.685} \\
\hline $40-69$ & $21(11 \%)$ & $12(10 \%)$ & $9(13 \%)$ & \\
\hline $70-119$ & $128(68 \%)$ & $83(70 \%)$ & $45(66 \%)$ & \\
\hline $120-159$ & $28(15 \%)$ & $19(16 \%)$ & $9(13 \%)$ & \\
\hline$\geq 160$ & $3(2 \%)$ & $2(2 \%)$ & $1(1 \%)$ & \\
\hline \multicolumn{5}{|l|}{ Lowest blood pressure } \\
\hline $\mathrm{MAP} \geq 70$ & $142(76 \%)$ & $97(82 \%)$ & $45(66 \%)$ & \multirow[t]{4}{*}{0.050} \\
\hline $\mathrm{MAP}<70$ & $33(18 \%)$ & $15(13 \%)$ & $18(26 \%)$ & \\
\hline Low dose vasopressor ${ }^{\mathrm{e}}$ & $4(2 \%)$ & $2(2 \%)$ & $2(3 \%)$ & \\
\hline High dose vasopressor $^{\mathrm{e}}$ & $6(3 \%)$ & $5(4 \%)$ & $1(1 \%)$ & \\
\hline
\end{tabular}

Bold values indicate statistical significance

Values shown are mean and SD, and number (\%). Categorical variables were tested using Fisher's exact test. Continuous variables were tested using the Wilcoxon rank sum test

${ }^{a}$ Among those admitted to the ICU $(n=105)$

${ }^{\mathrm{b}}$ Based on Quan et al. [15]

${ }^{\mathrm{c}}$ Among those with first admission to the hospital between January 1, 2016 and December 31, 2016 $(n=181)$

$\mathrm{d}_{38-41 \%}$ Data missing

${ }^{\mathrm{e}}$ Low dose defined as dopamine $\leq 15$ or epinephrine $\leq 0.1$ or norepinephrine $\leq 0.1 \mathrm{mcg} / \mathrm{kg} / \mathrm{min}$, and high dose defined as dopamine $>15$ or epinephrine $>0.1$ or norepinephrine $>0.1 \mathrm{mcg} / \mathrm{kg} / \mathrm{min}$

included age, use of home oxygen, Charlson co-morbidity index, diagnosis of peripheral vascular disease and hospital readmission. Age and hospital readmission are both factors that will obviously increase risk of death. The Charlson comorbidity index has been designed and validated as a predictor of death [15], and so this was also to be expected. The fact that peripheral vascular disease - a risk factor for 30 day readmission-was also a risk factor for death in patients with HRF was unexpected. We do not know the reason that peripheral vascular disease increases the risk of adverse outcomes related to HRF, though certainly it is a risk factor for readmission related to cardiovascular disease as noted above. It is possible that the combination of vascular insufficiency and respiratory insufficiency is a physiological combination that particularly increases risk of poor outcomes. Another interesting observation was the fact that use of chronic home oxygen was associated with increased risk of death. It is certainly possible that use of home oxygen is a marker of more advanced disease, but it is conceivable that home oxygen in patients with HRF could actually be harmful, as it may mask worsening disease related to hypercapnia, and even exacerbate hypercapnia through worsened ventilation:perfusion matching [29].

There are some limitations to the current data. This was a single center investigation which may limit generalizability, but does improve the internal validity of the study as data 
Fig. 1 Kaplan-Meier showing actual and $95 \%$ CI for survival of patients hospitalized with hypercapnic respiratory failure

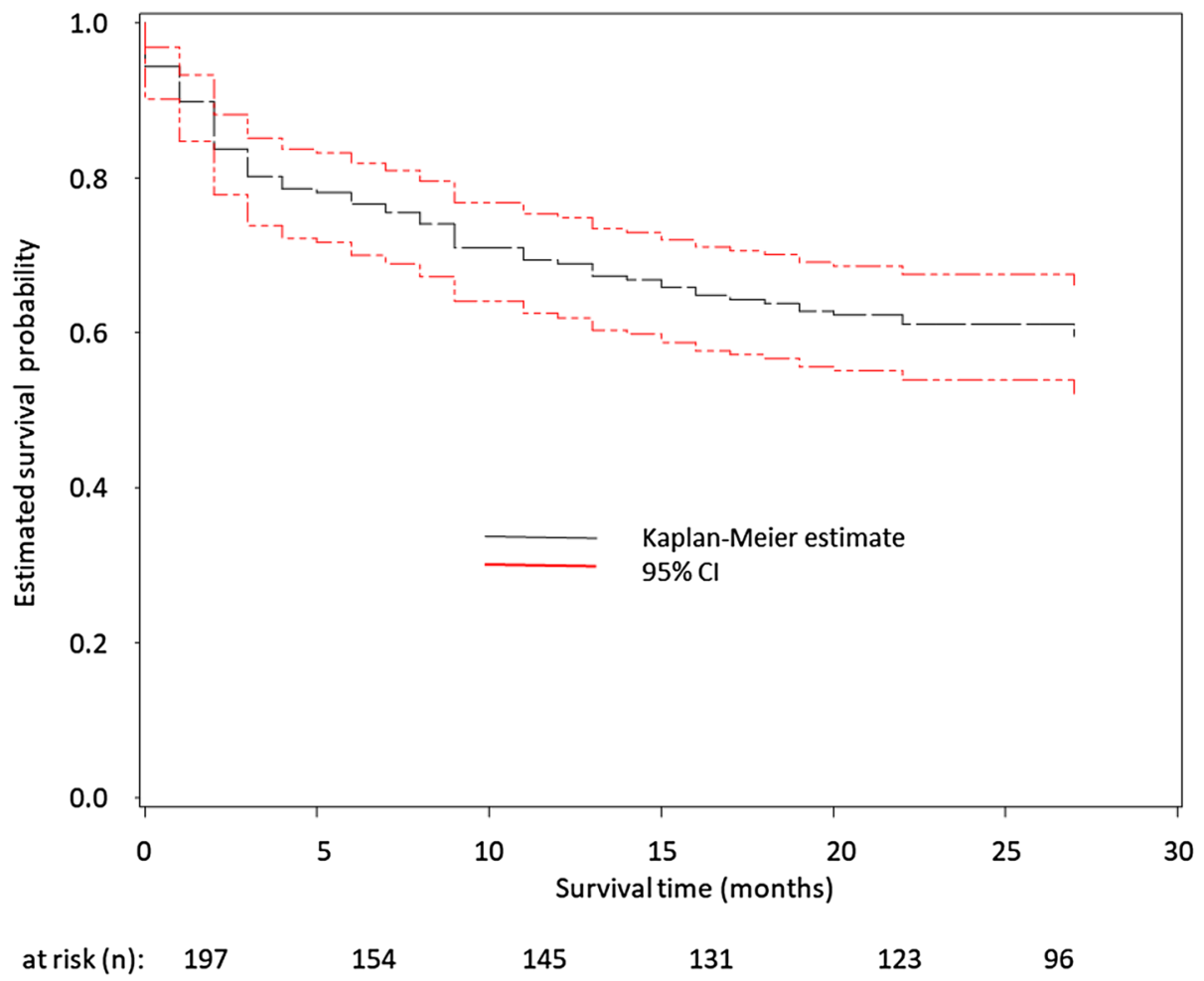

33

available via the electronic medical record were consistent across patients - our findings provide the basis for future studies at multiple sites. Another limitation is that patients were identified using ICD 10.0 codes for HRF; patients who were misidentified with HRF could have been included, others not identified with HRF would have been missed and not included in our analysis. However, as we reviewed all the medical records and excluded participants we felt had other reasons for HRF (such as cardiac arrest, seizure or neuromuscular disease) or limited life expectancy (related to advanced cancer, or conditions that in the opinion of the investigator would limit life expectancy to less than three months), this should be pertinent to patients suffering with HRF that would otherwise be expected to survive. On review of the electronic record, the authors felt that $80 \%$ of the deaths and admissions were definitely or possibly related to hypercapnic respiratory failure.
In conclusion, our data suggest that mortality is high in patients when admitted to the hospital with HRF, there is a high rate of 30 day readmission, and a high mortality rate in the months after discharge. Our data suggest that this patient population might require particularly careful follow-up following their index admission with interventions that might reduce the risk of readmission, such as pulmonary rehabilitation. Admission with HRF is a marker of patients at risk of poor outcomes. Certain factors are associated with particularly poor outcomes such as increased age, increased chronic comorbidities, and the presence of peripheral vascular disease. It is notable that use of home oxygen was associated with an increased risk of death-independent of other factors - and that few patients were treated with CPAP or BIPAP and none with NIV: it is possible that oxygen alone may actually be harmful in this patient population. 
Table 5 Unadjusted and adjusted odds ratios (and 95\% confidence intervals) from logistic regression analyses predicting death after discharge $(n=187)$

\begin{tabular}{|c|c|c|c|c|}
\hline & Unadjusted OR & $95 \% \mathrm{CI}$ & Adjusted OR & $95 \% \mathrm{CI}$ \\
\hline Sex: male vs. female & 0.895 & $(0.491,1.633)$ & & \\
\hline Age (by 5-year unit) & 1.333 & $(1.166,1.524)$ & 1.316 & $(1.125,1.538)$ \\
\hline BMI & 0.987 & $(0.966,1.010)$ & & \\
\hline Active smoker: (yes vs. no) & 0.371 & $(0.182,0.754)$ & & \\
\hline Length of admission (days) & 0.959 & $(0.926,0.994)$ & & \\
\hline \multicolumn{5}{|l|}{ Intensive care unit admission } \\
\hline (Yes vs. no) & 0.742 & $(0.408,1.352)$ & & \\
\hline Intubation (yes vs. no) ${ }^{\mathrm{a}}$ & 0.280 & $(0.126,0.620)$ & & \\
\hline \multicolumn{5}{|l|}{ Primary admission reason } \\
\hline Respiratory failure (yes vs. no) & 1.474 & $(0.810,2.683)$ & & \\
\hline Pneumonia (yes vs. no) & 1.189 & $(0.460,3.070)$ & & \\
\hline COPD exacerbation (yes vs. no) & 1.970 & $(0.986,3.936)$ & & \\
\hline Overdose (yes vs. no) & 0.420 & $(0.087,2.040)$ & & \\
\hline Sepsis (yes vs. no) & 0.418 & $(0.148,1.175)$ & & \\
\hline Trauma (yes vs. no) & 0.429 & $(0.047,3.919)$ & & \\
\hline Cardiac disease (yes vs. no) & 2.632 & $(1.368,5.062)$ & & \\
\hline \multicolumn{5}{|l|}{ Comorbidities } \\
\hline Myocardial infarction (yes vs. no) & 1.721 & $(0.714,4.144)$ & & \\
\hline Congestive heart failure (yes vs. no) & 3.124 & $(1.622,6.018)$ & & \\
\hline Peripheral vascular disease (yes vs. no) & 11.289 & $(2.421,52.634)$ & 12.562 & $(2.348,67.208)$ \\
\hline Cerebrovascular disease (yes vs. no) & 2.781 & $(0.756,10.228)$ & & \\
\hline Chronic pulmonary disease (yes vs. no) & 2.001 & $(1.053,3.801)$ & & \\
\hline Diabetes with chronic complications (yes vs. no) & 0.709 & $(0.358,1.405)$ & & \\
\hline Renal disease (yes vs. no) & 2.020 & $(0.968,4.217)$ & & \\
\hline \multicolumn{5}{|l|}{ Pulmonary disease } \\
\hline COPD (yes vs. no) & 1.940 & $(1.061,3.548)$ & & \\
\hline Obesity hyperventilation (yes vs. no) & 1.823 & $(0.564,5.891)$ & & \\
\hline Asthma (yes vs. no) & 0.671 & $(0.247,1.820)$ & & \\
\hline Interstitial lung disease (yes vs. no) & 5.446 & $(0.555,53.420)$ & & \\
\hline Bronchiectasis (including CF) (yes vs. no) & $<0.001$ & $(<0.001,>999)$ & & \\
\hline \multicolumn{5}{|l|}{ Home respiratory support } \\
\hline CPAP (yes vs. no) & 0.581 & $(0.217,1.552)$ & & \\
\hline BIPAP (yes vs. no) & 1.850 & $(0.661,5.177)$ & & \\
\hline Oxygen & 2.931 & $(1.572,5.464)$ & 4.029 & $(1.893,8.573)$ \\
\hline None & 0.503 & $(0.275,0.920)$ & & \\
\hline Charlson comorbidity index ${ }^{b}$ & 1.479 & $(1.208,1.811)$ & 1.388 & $(1.094,1.760)$ \\
\hline \multicolumn{5}{|l|}{ Discharge respiratory support } \\
\hline CPAP (yes vs. no) & 1.270 & $(0.387,4.167)$ & & \\
\hline BIPAP (yes vs. no) & 1.537 & $(0.626,3.774)$ & & \\
\hline Oxygen (yes vs. no) & 2.308 & $(1.253,4.251)$ & & \\
\hline None (yes vs. no) & 0.423 & $(0.229,0.783)$ & & \\
\hline \multicolumn{5}{|c|}{ Admission or death due to hypercapnic respiratory failure } \\
\hline Yes vs. no & 1.387 & $(0.639,3.010)$ & & \\
\hline Maybe vs. no & 1.250 & $(0.446,3.500)$ & & \\
\hline \multicolumn{5}{|l|}{ Readmission } \\
\hline Yes vs. no & 3.312 & $(1.753,6.257)$ & 3.066 & $(1.461,6.433)$ \\
\hline Days until first readmission ${ }^{\mathrm{c}}$ & 0.999 & $(0.995,1.003)$ & & \\
\hline Number of readmissions ${ }^{c}$ & 0.937 & $(0.767,1.144)$ & & \\
\hline
\end{tabular}


Table 5 (continued)

\begin{tabular}{|c|c|c|c|c|}
\hline & Unadjusted OR & $95 \% \mathrm{CI}$ & Adjusted OR & $95 \% \mathrm{CI}$ \\
\hline \multicolumn{5}{|c|}{ Readmission due to hypercapnic respiratory failure } \\
\hline Yes vs. no & 3.050 & $(1.232,7.551)$ & & \\
\hline Maybe vs. no & 0.639 & $(0.113,3.606)$ & & \\
\hline N/A vs. no & 0.934 & $(0.367,2.373)$ & & \\
\hline \multicolumn{5}{|l|}{ Bicarbonate } \\
\hline$\leq 19$ vs. $20-29$ & 1.413 & $(0.326,6.116)$ & & \\
\hline $30-39$ vs. $20-29$ & 2.521 & $(1.292,4.916)$ & & \\
\hline $40-49$ vs. $20-29$ & 7.536 & $(1.841,30.854)$ & & \\
\hline \multicolumn{5}{|c|}{ Hemodynamics in first $24 \mathrm{~h}$} \\
\hline \multicolumn{5}{|c|}{ Most extreme heart rate in first $24 \mathrm{~h}$} \\
\hline$\leq 69$ vs. $70-119$ & 1.537 & $(0.616,3.835)$ & & \\
\hline$\geq 120$ vs. $70-119$ & 0.878 & $(0.381,2.026)$ & & \\
\hline Other vs. MAP $\geq 70$ & 2.058 & $(1.027,4.121)$ & & \\
\hline
\end{tabular}

Bold values indicate statistical significance

$O R$ odds ratio, $C I$ confidence interval

${ }^{\mathrm{a}}$ Among those admitted to the ICU $(n=105)$

${ }^{\mathrm{b}}$ Based on Quan et al. [15]

${ }^{\mathrm{c}}$ Among those readmitted $(n=98)$

Author Contributions AJM and MCB were involved in study design, data collection, drafting and revision of the manuscript. JP was involved with data analysis and revision of the manuscript. CTB was involved in study design, data analysis and revision of the manuscript. AED was involved in study design, data collection, analysis, drafting and revision of the manuscript.

Funding This research did not receive any specific grant from funding agencies in the public, commercial, or not-for-profit sectors.

\section{Compliance with Ethical Standards}

Conflict of interest The authors have no conflicts of interest to report.

\section{References}

1. Adler D, Pepin JL, Dupuis-Lozeron E, Espa-Cervena K, MerletViolet R et al (2017) Comorbidities and subgroups of patients surviving severe acute hypercapnic respiratory failure in the intensive care unit. Am J Respir Crit Care Med 196(2):200-207

2. Marin JM, Soriano JB, Carrizo SJ, Boldova A, Celli BR (2010) Outcomes in patients with chronic obstructive pulmonary disease and obstructive sleep apnea: the overlap syndrome. Am J Respir Crit Care Med 182(3):325-331

3. U.S.B.o.D. Collaborators, Mokdad AH, Ballestros K, Echko M, Glenn S et al (2018) The state of US health, 1990-2016: burden of diseases, injuries, and risk factors among US states. JAMA 319(14):1444-1472

4. Senaratna CV, Perret JL, Lodge CJ, Lowe AJ, Campbell BE et al (2017) Prevalence of obstructive sleep apnea in the general population: a systematic review. Sleep Med Rev 34:70-81

5. Iftikhar IH, Roland J (2018) Obesity hypoventilation syndrome. Clin Chest Med 39(2):427-436
6. Soler X, Gaio E, Powell FL, Ramsdell JW, Loredo JS et al (2015) High prevalence of obstructive sleep apnea in patients with moderate to severe chronic obstructive pulmonary disease. Ann Am Thorac Soc 12(8):1219-1225

7. Ahmad N, Taithongchai A, Sadiq R, Mustfa N (2012) Acute hypercapnic respiratory failure (AHRF): looking at long-term mortality, prescription of long-term oxygen therapy and chronic non-invasive ventilation (NIV). Clin Med (Lond) 12(2):188

8. Chu CM, Chan VL, Lin AW, Wong IW, Leung WS et al (2004) Readmission rates and life threatening events in COPD survivors treated with non-invasive ventilation for acute hypercapnic respiratory failure. Thorax 59(12):1020-1025

9. Fazekas AS, Aboulghaith M, Kriz RC, Urban M, Breyer MK et al (2018) Long-term outcomes after acute hypercapnic COPD exacerbation: first-ever episode of non-invasive ventilation. Wien Klin Wochenschr 130(19-20):561-568

10. Simonds AK (2017) The road not taken: missed opportunities in managing acute exacerbations of hypercapnic respiratory failure. Am J Respir Crit Care Med 196(2):124-125

11. Konishi M, Akiyama E, Suzuki H, Iwahashi N, Maejima N et al (2015) Hypercapnia in patients with acute heart failure. ESC Heart Fail 2(1):12-19

12. Laserna E, Sibila O, Aguilar PR, Mortensen EM, Anzueto A et al (2012) Hypocapnia and hypercapnia are predictors for ICU admission and mortality in hospitalized patients with communityacquired pneumonia. Chest 142(5):1193-1199

13. Iqbal N, Irfan M, Zubairi ABS, Awan S, Khan JA (2017) Association of hypercapnia on admission with increased length of hospital stay and severity in patients admitted with community-acquired pneumonia: a prospective observational study from Pakistan. BMJ Open 7(6):e013924

14. Charlson ME, Pompei P, Ales KL, MacKenzie CR (1987) A new method of classifying prognostic comorbidity in longitudinal studies: development and validation. J Chronic Dis 40(5):373-383

15. Quan H, Li B, Couris CM, Fushimi K, Graham P et al (2011) Updating and validating the Charlson comorbidity index and score 
for risk adjustment in hospital discharge abstracts using data from 6 countries. Am J Epidemiol 173(6):676-682

16. Harris PA, Taylor R, Thielke R, Payne J, Gonzalez N et al (2009) Research electronic data capture (REDCap) - a metadata-driven methodology and workflow process for providing translational research informatics support. J Biomed Inform 42(2):377-381

17. Hosmer DW, Lemeshow S, Sturdivant RX (2013) Applied logistic regression. Wiley series in probability and statistics, 3 edn. Wiley, Hoboken

18. Lemyze M, Taufour P, Duhamel A, Temime J, Nigeon O et al (2014) Determinants of noninvasive ventilation success or failure in morbidly obese patients in acute respiratory failure. PLoS ONE 9(5):e97563

19. Goto T, Faridi MK, Gibo K, Toh S, Hanania NA et al (2017) Trends in 30-day readmission rates after COPD hospitalization, 2006-2012. Respir Med 30:92-97

20. Lau CS, Siracuse BL, Chamberlain RS (2017) Readmission after COPD exacerbation scale: determining 30-day readmission risk for COPD patients. Int J Chronic Obstr Pulm Dis 12:1891-1902

21. Angraal S, Khera R, Zhou S, Wang Y, Lin Z et al (2018) Trends in 30-day readmission rates for Medicare and non-Medicare patients in the era of the Affordable Care Act. Am J Med 131(11):13241331.e14

22. Beuther DA, Sutherland ER (2007) Overweight, obesity, and incident asthma: a meta-analysis of prospective epidemiologic studies. Am J Respir Crit Care Med 175(7):661-666

23. Garg J, Patel B, Chaudhary R, Shah M, Gupta R et al (2019) Predictors of 30-day readmissions after catheter ablation for atrial fibrillation in the USA. J Interv Card Electrophysiol 55(3):243-250
24. Vidula MK, McCarthy CP, Butala NM, Kennedy KF, Wasfy JH et al (2018) Causes and predictors of early readmission after percutaneous coronary intervention among patients discharged on oral anticoagulant therapy. PLoS ONE 13(10):e0205457

25. Kheirbek RE, Wojtusiak J, Vlaicu SO, Alemi F (2016) Lack of evidence for racial disparity in 30-day all-cause readmission rate for older US Veterans hospitalized with heart failure. Qual Manag Health Care 25(4):191-196

26. Basnet S, Zhang M, Lesser M, Wolf-Klein G, Qiu G et al (2018) Thirty-day hospital readmission rate amongst older adults correlates with an increased number of medications, but not with Beers medications. Geriatr Gerontol Int 18(10):1513-1518

27. Murphy PB, Rehal S, Arbane G, Bourke S, Calverley PMA et al (2017) Effect of home noninvasive ventilation with oxygen therapy vs oxygen therapy alone on hospital readmission or death after an acute COPD exacerbation: a randomized clinical trial. JAMA 317(21):2177-2186

28. Kohnlein T, Windisch W, Kohler D, Drabik A, Geiseler J et al (2014) Non-invasive positive pressure ventilation for the treatment of severe stable chronic obstructive pulmonary disease: a prospective, multicentre, randomised, controlled clinical trial. Lancet Respir Med 2(9):698-705

29. Abdo WF, Heunks LM (2012) Oxygen-induced hypercapnia in COPD: myths and facts. Crit Care 16(5):323

Publisher's Note Springer Nature remains neutral with regard to jurisdictional claims in published maps and institutional affiliations. 